\title{
ZUR DISKUSSION
}

DDS - Die Deutsche Schule

111. Jahrgang 2019, Heft 1, S. 103-119

https://doi.org/10.31244/dds.2019.01.10

(C) 2019 Waxmann

Isabell van Ackeren, Stefan Aufenanger, Birgit Eickelmann, Steffen Friedrich, Rudolf Kammerl, Julia Knopf, Kerstin Mayrberger, Heike Scheika, Katharina Scheiter \& Mandy Schiefner-Rohs

\section{Digitalisierung in der Lehrerbildung}

\section{Herausforderungen, Entwicklungsfelder und Förderung von Gesamtkonzepten}

\section{Zusammenfassung}

Auf Einladung des Bundesministeriums für Bildung und Forschung (BMBF) hat sich im Sommer 2018 eine bundesweite Expertengruppe mit zentralen Herausforderungen im Bereich der Digitalisierung in der Lehrerbildung beschäftigt. Auf der Grundlage der Einschätzung von Expert*innen sowie weiterer wissenschaftlicher Befunde und Erkenntnisse wurde vor diesem Hintergrund - auch unter Berücksichtigung der finanziellen Rahmenbedingungen - ein Konzept entworfen, wie eine entsprechende Förderlinie im Rahmen der Qualitätsoffensive Lehrerbildung aussehen könnte. Auch wurde beraten, welche Anforderungen an die Anträge im Sinne zukunftsweisender Gesamtkonzepte $z u$ stellen sind. Der Beitrag stellt insofern ein vertiefendes Hintergrundpapier zur Förderrichtlinie des BMBF in der Qualitätsoffensive Lehrerbildung mit dem Schwerpunkt Digitalisierung dar und eröffnet gleichzeitig Perspektiven für zukünftige Entwicklungen.

Schlüsselwörter: Digitalisierung, Lehrerbildung, Desiderata, Entwicklungsfelder, Förderung

\section{Digitization in Teacher Education}

Challenges, Development Areas, and Funding Objectives

\section{Summary}

Responding to the invitation of the Federal Ministry of Education and Research (Bundesministerium für Bildung und Forschung - BMBF), a nationwide expert group discussed key challenges in the area of digitization in teacher education in the summer of 2018. Against this background, based on further scientific findings and with regard 
to the financial conditions, it was considered what the corresponding funding line within the program "Qualitätsoffensive Lehrerbildung" could look like. The group also discussed the requirements for the applications in the sense of future-oriented overall concepts. In this respect, the article presents an in-depth background paper on the funding guidelines of the BMBF in the "Qualitätsoffensive Lehrerbildung" with a focus on digitization and offers perspectives for future developments.

Keywords: digitization, teacher education, desiderata, fields of development, promotion

Im Koalitionsvertrag vom 12. März 2018 der Bundesregierung in der aktuellen, 19. Legislaturperiode (Bundesregierung, 2018) wird mit einer eigenen Formulierung eine zusätzliche Schwerpunktsetzung der „Qualitätsoffensive Lehrerbildung“ auf den Bereich der Digitalisierung in der Lehrerbildung gelegt. Daran anknüpfend beschloss am 29. Juni 2018 die Gemeinsame Wissenschaftskonferenz (GWK) eine zusätzliche Auswahlrunde im Rahmen des Förderprogramms zu den beiden Themenbereichen Digitalisierung sowie Lehrerausbildung für die beruflichen Schulen. Notwendig wurde eine erneute Thematisierung, da entgegen der besonderen Relevanz beider vorgenannten Themenbereiche im Rahmen der Evaluation der ersten Phase der Qualitätsoffensive betont wurde, dass diese vergleichsweise wenig thematisiert wurden:

„Herausforderungen und Themenfelder, die das Förderprogramm bisher zu wenig adressiert hat, sind Digitalisierung in der Lehrerbildung, besondere Anforderungen im beruflichen Lehramt [...]" (Brümmer, Durdel, Fischer-Münnich, Fittkau, Weiger \& Altrichter, 2018, S. 8).

Um das für das Bundesministerium für Bildung und Forschung (BMBF) besonders relevante bildungspolitische Handlungsfeld „Digitalisierung“ in seinen Implikationen für die Lehrerbildung zu diskutieren und auf der Grundlage eines abgestimmten Konzeptes mögliche Handlungsbedarfe $\mathrm{zu}$ sondieren, fand im Sommer 2018 auf Einladung des BMBF ein Fachgespräch statt. Ziel war es, mit in Fragen der Digitalisierung in der Lehrerbildung ausgewiesenen Expert*innen sowie mit Vertreterinnen des BMBF unter Begleitung des Projektträgers DLR zukunftsweisende, bedarfsorientierte und auf Profilierung zielende Förderansätze für dieses Handlungsfeld zu diskutieren.

An dem Arbeitsgespräch nahmen die Autor*innen dieses Beitrags teil, die sowohl die Perspektive des Wissenschaftskontextes aus der Medienpädagogik, der Mediendidaktik, der Schulpädagogik, der Schulentwicklungsforschung, der Empirischen Lehr-Lernforschung und einzelner Fachdidaktiken einbrachten als auch die Perspektive des Bundesarbeitskreises der Seminar- und Fachleiter ${ }^{*}$ innen als Interessenvertretung aller in der Zweiten Phase der Lehrerausbildung Beschäftigten vertraten. Im Rahmen dieses Fachaustauschs entstand vor dem Hintergrund der Vorbereitung 
der neuen Förderrichtlinie der „Qualitätsoffensive Lehrerbildung“ ${ }^{1}$ das vorliegende Papier. Es skizziert zentrale Herausforderungen der Digitalisierung für die Lehrerbildung, identifiziert mögliche Entwicklungsfelder und Förderziele und stellt Desiderata für die künftige Förderpolitik zusammen.

\section{Ausgangslage und Herausforderungen}

Der gesellschaftliche Wandel in einer digitalisierten, vernetzten und automatisierten Welt und die mit einer Digitalisierung verbundenen Transformationsprozesse gehen mit Fragen nach systematischen Veränderungsbedarfen und -potenzialen, die neben anderen Bereichen wie Ökonomie und Recht auch und insbesondere im Bildungsbereich wichtig zu stellen und zu klären sind, einher. Dieses betrifft gleichermaßen eine technologische, eine gesellschaftlich-kulturelle sowie eine anwendungsbezogene Perspektive.

Dabei hat Schule als Ort formaler Bildungsprozesse für alle Kinder und Jugendlichen eine besondere Bedeutung. Ihr kommt die Aufgabe zu, systematisch unterschiedliche Modi des Verstehens und Handelns in einer durch Digitalisierung geprägten Welt zu fördern, um Orientierung zu geben und möglichen Bildungsdisparitäten entgegenzuwirken. Dies ist auch vor dem Hintergrund zu sehen, dass insbesondere im Bereich der Digitalisierung dem außerschulisch und informell geprägten Kompetenzerwerb eine besondere Rolle zukommt, da dieser mit sozialen Herkunftsmerkmalen von Kindern und Jugendlichen korrespondiert und Schule hier eine ausgleichende Funktion übernehmen sollte. Diesbezüglich konnten Studien wie etwa ICILS 2013 (International Computer and Information Literacy Study; Bos et al., 2014) für Deutschland aufzeigen, dass Bildungschancen auch in diesem zukunftsweisenden Bereich ungleich verteilt sind und von der sozialen Herkunft, vom Geschlecht sowie vom Migrationshintergrund der Schülerinnen abhängen.

\subsection{Aufgabe des Bildungssystems}

Für den schulischen Bildungsbereich ergibt sich daraus die Aufgabe, der digitalen Spaltung aktiv und durch systematische Verankerung des schulischen Lehrens und Lernens über digitale, mit digitalen und trotz digitaler Medien (Döbeli Honegger, 2016) gezielt entgegenzuwirken. Weitere im internationalen Vergleich besondere Herausforderungen des Entwicklungsbedarfs computer- und informationsbezogener Kompetenzen von Schüler*innen in Deutschland sowie Handlungsbedarfe im Hinblick auf die lernförderliche Nutzung digitaler Medien zeigen sich nicht zu-

1 Vgl. die entsprechende Bekanntmachung unter URL: https://www.bmbf.de/foerderungen/ bekanntmachung-2097.html. 
letzt ebenfalls auf empirischer Basis im Rahmen von ICILS 2013 (Bos et al., 2014). Darüber hinaus weisen Studien wie etwa der Länderindikator „Schule digital“ auf die besondere Notwendigkeit einer zeitgemäßen Weiterentwicklung aller Phasen der Lehrerbildung hin (Eickelmann, Lorenz \& Endberg, 2016).

Der kompetente rezipierende, produktive, kritische, kreative und zielgerichtete Umgang mit digitalen Medien wird in einem breiten Begriffsverständnis - nicht nur im Strategiepapier „Bildung in der digitalen Welt“ der Kultusministerkonferenz (KMK, 2016) als eine neue Kulturtechnik beschrieben (vgl. auch Schelhowe et al., 2009), die neben die traditionellen Kulturtechniken tritt, diese aber zugleich auch verändert (KMK, 2016). Diese Veränderungen betreffen verschiedene Facetten und Verantwortungsbereiche in Bildung und Schule (vgl. Friedrich, 2017). Lehrkräfte sind durch damit eingehergehende tiefgreifende Entwicklungen besonders herausgefordert, sich sehr grundsätzlich, zukunftsweisend und verantwortlich mit Bildung und der Gestaltung von Lehr- und Lernprozessen in der digitalen Welt auseinanderzusetzen.

Dafür benötigen Lehrkräfte eine für Veränderungen und Innovationen, aber auch für Ungewissheit offene Haltung und Kompetenzen, die nicht nur auf bestehende Wissensbestände, sondern auf reflektierte Flexibilität setzen. In diesem Sinne müssten Lehrkräfte zukünftig stärker als bisher fähig sein bzw. befähigt werden, digitale Medien kompetent und didaktisch reflektiert für die (fachspezifische) Gestaltung von Lehren und Lernen im Kontext der Organisation Schule - auch im Hinblick auf eine Kultur des Teilens - einzusetzen und Kompetenzen für eine zielgerichtete Orientierungs- und Handlungsfähigkeit der Schüler*innen in der digital geprägten Gesellschaft zu fördern.

Digitalisierung von Schule und Unterricht ist dabei kein Selbstzweck, sondern so anzulegen, dass insbesondere pädagogische Ziele verfolgt werden, um Schüler ${ }^{\star}$ innen angemessen auf das Leben in der derzeitigen und künftigen Gesellschaft vorzubereiten und sie damit zur aktiven und verantwortlichen Teilhabe am kulturellen, gesellschaftlichen, politischen, beruflichen und wirtschaftlichen Leben zu befähigen (Cress et al., 2018). In diesem Zusammenhang unterliegt die Perspektive auf das Lehren und Lernen grundsätzlichen Veränderungen, etwa im Hinblick auf die Möglichkeiten

- räumlich und zeitlich entgrenzten Lernens,

- adaptiven und individualisierten Lernens,

- kooperativer und kollaborativer Lernsettings,

- kognitiver Aktivierung sowie

- problem- und handlungsorientierten Lernens,

die stärker selbstgesteuerte und partizipative Lernphasen ermöglichen (Scheiter, 2017). Zugleich erfordern diese auch eine Veränderung der Tätigkeit und Rollen von Lehrer*innen sowie von Schulleitungen (Eickelmann \& Gerick, 2017). Zugleich 
fordert eine sich in dieser Form entwickelnde Lehr- und Lernkultur auch mehr Bereitschaft von Seiten der Schüler ${ }^{\star}$ innen, von Beginn an zunehmend Verantwortung für den eigenen Lernprozess zu übernehmen. Dabei können digitale Bildungsmedien im weitesten Sinne die Lernmöglichkeiten erweitern und digitale Medien insbesondere bei organisatorischen und kommunikativen Interaktionsprozessen unterstützen.

Dabei verändern sich auch die wissenschaftlichen Bezugsdisziplinen der Unterrichtsfächer bzw. der beruflichen Fachrichtungen durch Digitalisierung stetig weiter, etwa im Hinblick auf die Generierung neuen Wissens und Möglichkeiten neuer Wissenserschließung, durch grundlegende Veränderungen von Prozessen und Vernetzung, z. B. durch die Verfügbarkeit großer Datensätze und die Verknüpfung von Information aus multiplen Quellen oder durch computergestützte Verarbeitungsmethoden in Form von Visualisierungen, Simulationen, aber auch Big Data.

Zugleich bleibt die vielfach verbreitete Annahme, dass die künftige Lehrergeneration durch ein Aufwachsen in der digitalen Welt quasi automatisch bereits über die dafür benötigten Kompetenzen verfüge, unerfüllt (Eickelmann, 2018). Deutlich wird, dass sich aus den beobachteten und zukünftigen Veränderungen neue Gestaltungs- und Handlungsbedarfe für die Lehrerbildung und Professionalisierung von Lehrkräften ergeben, die nur sehr bedingt durch die bisherigen Ansätze und Strukturen der Lehrerbildung bearbeitet werden können (vgl. Schiefner-Rohs et al., 2018).

\subsection{Zukunftsweisende Lehrerbildung}

Ziel einer zukunftsweisenden Lehrerbildung muss es sein, alle in Schule Tätigen so $\mathrm{zu}$ qualifizieren und darüber hinaus bereits in der Lehrerausbildung entsprechend vorzubereiten, dass sie sich mit stetig wandelnden Anforderungen auseinandersetzen und Potenziale für neue Lernwege erschließen, sie reflektieren und Kompetenzen erwerben, diese stetig weiterzuentwickeln. Hierbei kommt sowohl den lehrerbildenden Hochschulen als forschenden und lehrenden Einrichtungen als auch den Institutionen der zweiten Lehrerausbildungsphase eine zentrale Rolle zu. Bereits hier müssen in dafür geeigneten Lernsettings entsprechende Kompetenzen im Sinne eines breiten Kompetenzbegriffs zunächst erworben und innerhalb der Lehrerbildungskette weiter professionalisiert werden, um später in der eigenen Schul- und Unterrichtsgestaltung reflektiert umgesetzt und kontinuierlich weiterentwickelt werden zu können.

Neben medienbezogenen Kompetenzen erscheint es mit Blick auf die eingangs aufgezeigte Perspektive auf umfassende Transformationsprozesse ebenso notwendig, die Förderung von reflexiven Prozessen im Sinne einer umfassenden Medienbildung mit (kritischem) Gesellschaftsbezug über die Lehrerbildungskette hinweg zu realisieren, um Digitalisierung und Digitalität (Stalder, 2018) auch als Lerngegenstand zu erweitern. 
Isabell van Ackeren et al.

Alle Einrichtungen der Lehrerbildung müssen die Entwicklung digitalisierungsbezogener Kompetenzen fördern, indem sie

- medienpädagogische Kompetenz (im Sinne von Wissen, Können, Reflexivität und berufsethischer Haltung, nicht nur im Hinblick auf Unterricht, sondern auch mit Blick auf Schule als Organisation),

- fachdidaktische Kompetenz (Veränderung/Erweiterung von Lerngegenständen und fachspezifisch zu fördernden Kompetenzen unter den Bedingungen von Digitalisierung und Digitalität) und

- informatische Kompetenz (im Sinne von algorithmischem Denken, Data Literacy, Computational Thinking und Datensicherheit)

in Hinblick auf Fachgegenstände sowie die Schule als Organisation im gesellschaftlichen Kontext integrieren und aufeinander beziehen. Daneben müssen Kompetenzen für den Einsatz innovativer mediendidaktischer Konzepte für die digitale Unterstützung von Lernprozessen - auch unter Berücksichtigung der Gestaltung organisationaler Rahmenbedingungen von Schule - erworben werden und die Rahmenbedingungen schulischen Lernens insgesamt wie auch hard- und softwaretechnische Grundlagen hinterfragt und an Praxisbeispielen reflektiert werden.

Alle lehrerausbildenden Einrichtungen der ersten, zweiten und dritten Phase der Lehrerbildung stehen dabei in einer besonderen Verantwortung, (angehende) Lehrkräfte entsprechend aus- und fortzubilden, um damit den spezifischen Wert digitaler Technologien und entsprechender Praktiken zielgerichtet für die Schul-, Unterrichtsund Lernentwicklung zukunftsorientiert nutzen zu können. Nachfolgende Generationen sollen bestmöglich auf die Anforderungen der sich durch Digitalisierung stetig ändernden Lebens- und Arbeitswelt vorbereitet werden. Dabei bietet sich auch die Chance für Hochschulen, ausgehend von der Entwicklung der Lehrerbildung Impulse für weitere Studiengänge zu setzen.

Die Kooperation mit der zweiten Phase im Sinne der Ermöglichung eines kumulativen Kompetenzaufbaus erscheint im Zuge dieser Entwicklungen unerlässlich. Zudem wird dem staatlichen Fortbildungssystem die Rolle der kontinuierlichen Sicherung und Aktualisierung der benötigten Kompetenzen zukommen, wie die Ergebnisse der ICILS-2013-Studie für im Bereich der Digitalisierung bereits erfolgreiche Schulsysteme aufzeigen.

\subsection{Bisherige Erfahrungen (nicht nur) in der Förderlinie}

Trotz der hervorgehobenen Bedeutung des Themas Digitalisierung in der Lehrerbildung fanden sich in der ersten Phase der "Qualitätsoffensive Lehrerbildung“ in den ersten beiden Antragsrunden nur wenige Projekte an teilnehmenden Hoch- 
schulen mit einer expliziten Schwerpunktsetzung im Bereich Digitalisierung oder der Etablierung mediengestützten Lernens, in denen es neue Lernangebote, neue Veranstaltungsformate und strukturelle Veränderungen im Hinblick auf die phasenübergreifende Kooperation gibt.

Die bereits oben angesprochene Zwischenevaluation des Programms „Qualitätsoffensive Lehrerbildung“ (Brümmer et al., 2018) zeigte zudem, dass sich die vorhandenen Ansätze digitalen Lernens und der Digitalisierung von Lehrerbildung in den Projekten vielfach auf die Themen Heterogenität und Inklusion bezogen und Digitalisierung demnach als Chance für adaptives, individualisiertes und inklusives Lernen in Schulen verstanden wurde. Diese Projekte zeigen, dass Lernprozesse räumlich und zeitlich flexibler - auch in der Verknüpfung von Schule und Hochschule - organisiert, individuelle Lernwege und Lerngeschwindigkeiten berücksichtigt, Selbstlernphasen durch vielfältige Materialien angereichert und kooperative LehrLernformen sowie Lernerfolgskontrollen integriert werden können. Darüber hinaus finden sich Bezüge zur Digitalisierung im Bereich des Einsatzes von Unterrichtsvideos und Videografie.

Allerdings wurden Medien und Digitalisierung als Lerngegenstände bislang kaum systematisch betrachtet, und die umfassenden Anforderungen und notwendigen Veränderungen in der Lehrerbildung vor dem Hintergrund des digitalen Wandels kommen unter Berücksichtigung der hohen und weiter steigenden Relevanz des Themas, auch vor dem Hintergrund der KMK-Strategie, bisher in Deutschland zu kurz. Eine umfassende, fächerübergreifende und fächerspezifische, medienbezogene bildungswissenschaftliche und informatische Kompetenzentwicklung ist in der Lehramtsausbildung bislang nicht systematisch und damit nicht verbindlich angelegt. Dabei kann auf erste Ansätze der Modellierung entsprechender Kompetenzen zurückgegriffen werden (vgl. u.a. Europäische Union, 2017) ${ }^{2}$. Dieses und weitere Kompetenzmodelle für die Lehrerbildung in der digitalen Welt werden derzeit zudem aus wissenschaftlicher und schulpraktischer Sicht bei der Entwicklung eigener Konzepte in der Lehrerbildung in den Bundesländern reflektiert und entwickelt (u.a. Forschungsgruppe Lehrerbildung Digitaler Campus Bayern, 2017; KMK, 2018).

2 Ein Vergleich mit internationalen Kompetenzmodellen zeigt, dass in deutschsprachigen Beiträgen der kritisch-reflexive Zugang eine stärkere Bedeutung hat. So hat der Europäische Referenzrahmen für digitale Kompetenzen (European Digital Competence Framework for Citizens - DigComp) fünf Kompetenzbereiche, die im KMK-Modell auch auftauchen (Kompetenzbereiche: „Suchen und Verarbeiten“, „Kommunizieren und Kooperieren“, „Produzieren und Präsentieren“, „Schützen und sicher agieren“, „Problemlösen und Handeln“). Darüber hinaus ist "Analysieren und Reflektieren“ ein eigener Bereich. Dies kann als Besonderheit des deutschsprachigen Verständnisses von „Bildung“ interpretiert werden. Im Anschluss an die Bildungstheorie, die auf ein reflexives Verhältnis zur Welt, zu den sozialen Anderen und zu sich selbst abzielt, hat diese Dimension einen zentraleren Stellenwert in der Bestimmung der Bildungsziele. Darüber hinaus mag auch die kollektive Erfahrung zweier Unrechtsregime, die u. a. mit der Kontrolle und Gleichschaltung der Massenmedien ihre Propaganda verbreiteten, dazu beigetragen haben. 
In seiner Zusammenschau kommt der Monitor Lehrerbildung in seiner 2018 vorgelegten Studie zu dem Schluss, dass insbesondere das Lehramtsstudium in den Bundesländern derzeit weit davon entfernt sei, Inhalte des Studiums zum Umgang mit digitalen Medien im Sinne der von der Kultusministerkonferenz angestrebten Ziele verbindlich festzuschreiben (Bertelsmann Stiftung, CHE Centrum für Hochschulentwicklung, Deutsche Telekom Stiftung \& Stifterverband für die Deutsche Wissenschaft, 2018). Entsprechend benötigte Angebote seien bisher nicht flächendeckend verfügbar, nicht zuletzt auch aufgrund fehlender Expertise bzw. fehlender thematisch einschlägiger Professuren in den Fachdidaktiken sowie in den Bildungswissenschaften an vielen Standorten der Lehrerausbildung.

Mit mehr Nachdruck als bisher gilt es daher, Digitalisierung als wichtige Querschnittsbzw. Integrationsaufgabe der Lehrerbildung im Hinblick auf alle Unterrichtsfächer, beruflichen Fachrichtungen bzw. Fächergruppen, die bildungswissenschaftlichen Anteile sowie hinsichtlich der Schulformen und Schulstufen, die einer curricularen Verankerung bedarf, einzubeziehen und die Kompetenzentwicklung systematisch - auch über die Ausbildungsphasen hinweg - zu befördern. Die Rolle der Informatik in der Lehrerbildung, etwa Kompetenzen im Hinblick auf algorithmisches Denken, zum Programmieren, zu Datensicherheit und Verschlüsselung, zur digitalen Kommunikation und Kooperation, zu Datenbanken oder auch Big Data und weiteren Themenfeldern, ist dabei ebenso einzubeziehen wie die Bedeutung der Informationsund Medienethik.

\section{Entwicklungsfelder}

Mit der zusätzlichen, thematisch fokussierten Förderrunde der „Qualitätsoffensive Lehrerbildung“ wird das Desiderat aufgegriffen, Digitalisierung als ein hoch relevantes Themenfeld der Lehrerbildung im Sinne eines eigenständigen Lernbereichs und als Querschnittsperspektive erstmals in den Fokus bundesweiter Förderung durch Bund und Länder zu rücken. Damit soll die Entwicklung hervorragender, innovativer Professionalisierungskonzepte systematisch gefördert werden, die zugleich das Potenzial haben, nicht nur innerhalb eines Faches bzw. einer beruflichen Fachrichtung sowie eines Standortes, sondern auch über einzelne Fächer und Standorte hinaus in die Breite zu wirken. Dabei ist die Transferierbarkeit der Ansätze als wichtiges Kriterium des Erfolges von Vorhaben in der Konzeptionierung mitanzulegen.

Über die entsprechende Professionalisierung der in der Lehrerausbildung Tätigen sowie insbesondere der angehenden Lehrer*innen werden letztlich die zukunftsfähige Entwicklung von Schule und Unterricht sowie die Kompetenzentwicklung der Schüler*innen und ihre Fähigkeiten zur Orientierung in und kompetenten Gestaltung 
von digitaler Welt in individueller und gesellschaftlicher Perspektive maßgeblich beeinflusst.

Auf der Grundlage der Analysen der Expertengruppe erscheinen hier derzeit nachfolgende Themen- und Handlungsfelder mit dem Ziel der Entwicklung exzellenter innovativer und nachhaltig wirkender Ansätze für lehrerausbildende Hochschulen zentral.

\subsection{Verzahnte Kompetenzentwicklung und curriculare Verankerung}

Eine zentrale Voraussetzung für eine verzahnte Kompetenzentwicklung ist die verbindliche Verankerung entsprechender Kompetenzziele, u.a. in technischer, didaktischer, unterrichtspraktischer und schulentwicklungsbezogener Hinsicht. Diese systematische Verankerung erfolgt dabei sinnvoll in allen Bereichen der Ausbildungscurricula im Sinne eines kontinuierlichen und kumulativen Kompetenzaufbaus in den Bildungswissenschaften sowie innerhalb der Fächer bzw. beruflichen Fachrichtungen, zwischen den Fächern im Sinne der Vernetzung der fachdidaktischen, fachwissenschaftlichen und bildungswissenschaftlichen Studienanteile, über die Ausbildungsphasen hinweg und zwischen den einzelnen Phasen der Lehrerbildung, insbesondere mit der zweiten Phase der Lehrerausbildung, aber auch denkbar in Koordination mit der Lehrerfortbildung und den staatlichen Fortbildungssystemen. Dabei sollten Lehr-, Lern- und Prüfungsprozesse aufeinander abgestimmt sein und die Lerngelegenheiten selbst die Möglichkeiten der Digitalisierung im Sinne des didaktischen Doppeldeckers (Geissler, 1985) sinnvoll und modellhaft (KMK, 2016) nutzen.

\subsection{Praxis- und Handlungsorientierung}

Um angehende Lehrkräfte bestmöglich auf ihre Aufgaben in einer digitalisierten Welt vorzubereiten, benötigen diese Lerngelegenheiten, die relevante wissenschaftliche Erkenntnisse einbeziehen. Vor allem sind aber auch praxisorientierte Lerngelegenheiten und entsprechende (positive) Erfahrungen, die die Schulwirklichkeit miteinbeziehen, die Berührungsängste nehmen, motivieren und Freiräume für Entwicklung und Erprobung ermöglichen, schon ab dem ersten Semester wichtig.

Die Kompetenzentwicklung ist gleichermaßen sowohl wissenschaftich fundiert als auch praxisrelevant $\mathrm{zu}$ gestalten. Einer entsprechenden Ausgestaltung der Lerngelegenheiten in der Hochschule, in den universitären Praxisphasen sowie in den Einrichtungen der zweiten und dritten Phase der Lehrerbildung kommt hierbei eine zentrale Funktion zu. Zu diesen praxisorientierten Lerngelegenheiten und Erfahrungsräumen gehören der infrastrukturelle und konzeptionelle Aufbau und 
die Ausgestaltung von physischen oder auch (teil-)virtuellen Lehr-Lern-Laboren an den Hochschulen. An die Hochschule angebundene, medientechnisch lernförderlich ausgestattete Klassenzimmer tragen mit entsprechenden Nutzungskonzepten zur Praxisintegration und Handlungsorientierung, auch an den Schnittstellen der unterschiedlichen Ausbildungsphasen der Lehrerbildung, bei. Dabei sind insbesondere auch standortübergreifende, datenschutzrechtlich einwandfreie Lösungen notwendig.

\subsection{Offene Bildungsressourcen}

Standortübergreifende sowie phasenübergreifende Lösungen betreffen zudem die Bereitstellung didaktisch aufbereiteter Konzepte einschließlich der verwendeten Lerninhalte in Form von Bildungsmedien auf zentralen, standortübergreifenden internetbasierten Lernplattformen (z.B. in Anbindung an die Entwicklungen zu OER am $\left.\mathrm{DIPF}^{3}\right)$. So werden Beispiele guter und zugleich wissenschaftlich fundierter Lehr- und Lernpraxis frei lizensiert und offen zugänglich dokumentiert, zugänglich gemacht und verbreitet - und dadurch zugleich auch eine Form von kooperativem und kollaborativem Lehren und Lernen bzw. eine Form der Lehrerkooperation gefördert.

Offene Bildungsressourcen sind daher nicht nur als Umgang mit Material zu begreifen, sondern implizieren gleichsam die Entwicklung einer kooperativen Haltung, indem insbesondere eine Kultur des Teilens im Sinne sog. offener Bildungspraktiken mitgedacht wird. Dies umfasst auch die Frage nach der Entwicklung und Erprobung kooperativer Lehrpraktiken, die vor dem Hintergrund von Digitalisierungsprozessen stärker als bisher Teil der Professionalisierung in der Lehrerbildung sein müssten.

Entsprechend bilden „Pools“ mit gut dokumentierten und adaptierbaren reflektierten Praxisbeispielen sowie Lerninhalte in Form von offenen und frei zugänglichen digitalen Bildungsmedien eine wichtige Ressource für die umfassende, praxisnahe Ausbildung von Lehrkräften im Sinne der „Praxis- und Handlungsorientierung“ und damit einen wichtigen Professionalisierungsbaustein.

\subsection{Professionalisierung der Lehrenden}

Die Förderung digitaler Kompetenzen in der Aus-, Fort- und Weiterbildung erfordert auch Konzepte zur (Weiter-)Qualifizierung von Lehrenden in allen Phasen der Lehramtsausbildung. Konsens ist, dass Lehrende selbst über einen hohen Professionalisierungsgrad in der Umsetzung der Anforderungen, die sich aus dem digitalen Wandel für die Lehrerbildung ergeben, verfügen müssen. Hierfür müssen -

3 Vgl. URL: https://www.dipf.de/de/forschung/aktuelle-projekte/informationsstelle-oer-infor mation-transfer-und-vernetzung-zu-open-educational-resources; Zugriff am 01.11.2018. 
gleichwohl nicht nur für die Lehrerausbildung, sondern für alle Studien-, Fort- und Weiterbildungsangebote - verlässliche Unterstützungssysteme verfügbar sein, die die Professionalisierung in technischer und didaktischer Hinsicht im Sinne von Beratung und Umsetzung der Konzepte für digitalbasierte Lernsettings gezielt und zuverlässig ermöglichen und begleiten.

Dabei erscheint von besonderer Relevanz, dass die Konzepte weit über die technischen Kompetenzen hinausgehen und Fragen von Medienbildung selbstverständlich mit einschließen. Perspektivisch gilt es, in der Lehreraus- und -fortbildung Kompetenzen zu vermitteln und zu fördern, die zu einer zukunftsfähigen Gestaltung von Schule und Unterricht unter den Bedingungen der Digitalisierung befähigen.

\subsection{Anreizstrukturen ausbauen}

Entsprechende Anreizstrukturen, schon bei der Personalgewinnung an den Hochschulen und Ausbildungsstandorten der zweiten und dritten Phase, einschließlich einer möglichst hohen Verbindlichkeit zur persönlichen Fortbildung und Professionalisierung im Bereich der digitalen Qualifikation, können dazu beitragen, Engagement im Kontext der Digitalisierung in der Lehrerbildung zu fördern und anzuerkennen. Dazu gehört vor allem auch eine Digitalisierungsstrategie der Hochschulen mit entsprechenden, auf die Lehrerbildung bezogenen Leitlinien, die die Relevanz des Themas Digitalisierung als prioritäres Aufgabenfeld auch aus Sicht der Leitungen aller Einrichtungen der Lehrerbildung hervorheben und eine Identitäts-, Identifikations- und Mobilisierungsfunktion haben.

Dies kann zum Beispiel in spezifischen Angeboten medien- und hochschuldidaktischer Fortbildungen für Hochschullehrende und wissenschaftliche Mitarbeiter*innen, in Berufungs- und Zielvereinbarungen sowie in Form von besonderen Lehrpreisen für den Einsatz digitaler Medien in der akademischen Lehre oder gesonderter Förderung mit Ressourcen von entsprechend ausgerichteten Lehrprojekten (z.B. in Lehr-Lern-Laboren) gestaltet werden. Nur so kann sichergestellt werden, dass die Lehrenden in der Lehrerbildung selbst digitale Medien so einsetzen, dass Lehramtsstudierende damit ein Modell für ihre eigene unterrichtliche Tätigkeit vorgestellt bekommen und entsprechendes Engagement gefördert und wertgeschätzt wird.

\subsection{Koordination und Kooperation}

Die Zentren für Lehrerbildung bzw. Schools of Education an den lehrerausbildenden Universitäten und Hochschulen sind auch für den Bereich der Digitalisierung zentrale Akteure an der Schnittstelle der verschiedenen Phasen und Kooperationspartner der Lehrerbildung. Sie nehmen - auch in der Vernetzung mit den Einrichtungen der 
| Isabell van Ackeren et al.

zweiten Phase der Lehrerausbildung sowie untereinander oder auch in Kooperation mit Pädagogischen Landesinstituten - eine wichtige Rolle bei der reflektierten Entwicklung von Digitalisierungsprozessen in der und für die Lehrerbildung ein. Für diese neuen Aufgabenfelder sind sie entsprechend $\mathrm{zu}$ unterstützen. Dies betrifft in gleicher Weise die Schaffung personeller und finanzieller Ressourcen für die verschiedenen Handlungsfelder sowie für die eigene Organisationsentwicklung, für die Übernahme neuer Aufgaben sowie für die Kooperationsaufgaben in der Zusammenarbeit mit den Studienseminaren und Landesinstituten.

Weitere wichtige Akteure und Kooperationen ergeben sich - insbesondere auch im Verbund - mit den Hochschulrechenzentren, den Bibliotheken, den hochschuldidaktischen Einrichtungen und Zentren für E-Learning oder Digitalisierung in der Lehre sowie den einschlägigen Professuren und Arbeitsbereichen. Kooperationen sind aber auch zwischen lehrerbildenden Universitäten weiterzuentwickeln und zu befördern. So können beispielsweise landesweite Einrichtungen und Initiativen (z. B. der Virtuelle Campus Rheinland-Pfalz (VCRP) oder die virtuelle Hochschule Bayern) zentrale Unterstützer sein, kooperative Netzwerke über Universitätsgrenzen hinweg aufzubauen und Synergien für die Lehrerbildung und den Transfer von Konzepten zu nutzen.

\subsection{Forschungsorientierung und Evaluation}

Maßnahmen sollten zukünftig stärker als bisher anschlussfähig an internationale Diskurse zu medien- und digitalisierungsbezogenen Kompetenzen und Kompetenzentwicklung sowie an entsprechende Forschung in den verschiedenen Disziplinen entwickelt werden und durch Evaluationen Prozesse und Ergebnisse von Veränderungen, etwa im Hinblick auf erreichte Kompetenzniveaus, aufzeigen und einordnen. In diesem Zusammenhang kommt der Nachwuchsförderung an Universitäten eine besondere Rolle zu. Zudem kann die Digitalisierung durch die Nutzung erweiterter Datenbestände einen Beitrag zur kontinuierlichen und qualitätsgesicherten Weiterentwicklung der universitären Lehramtsausbildung leisten, etwa im Rahmen von Learning Analytics sowie durch die Integration in Qualitätsmanagementsysteme.

\section{Förderung zukunftsweisender Gesamtkonzepte}

Die aktuelle, zusätzliche Förderrunde zur Digitalisierung im Rahmen der „Qualitätsoffensive Lehrerbildung“ erscheint vor dem Hintergrund der hier vorgestellten Analysen als ein sehr gutes Signal und ein Schritt in die richtige Richtung. Sie kann aber, schon angesichts ihres übersichtlichen finanziellen Rahmens, nur ein Anfang sein und erste Impulse für die Qualitätsentwicklung der Lehrerbildung in der digi- 
talen Welt geben. Diese und weitere Programmförderungen bieten gleichwohl die Chance, die Entwicklung und Sichtbarmachung

- besonders innovativer und zukunftsweisender Entwicklungsmodelle

- mit einer klaren Vision für den Transformationsprozess der Lehrerbildung und

- für die zukunftsfähige Kompetenzentwicklung möglichst vieler (angehender) Lehrkräfte

im Bereich der Digitalisierung in der Lehrerausbildung zu unterstützen. Von der Digitalisierung ausgehende Veränderungsprozesse in der Lehrerausbildung sollten dabei so konzeptioniert werden, dass sie als ein profilbildendes Element in die Hochschulen hinein wirken und bei den Einrichtungen der beteiligten Projektpartner nachhaltig sichtbar werden und den Transfer der Ergebnisse ermöglichen. Die Förderung sollte daher vor allem die Realisierung von Konzepten ermöglichen, die $\mathrm{zu}$ einer standortübergreifenden inhaltlichen und strukturellen Profilierung des Themenfeldes beitragen und letztlich die Entwicklung der Fähigkeiten, Fertigkeiten, Einstellungen und Haltungen angehender Lehrkräfte befördern.

Somit geht es in einem bundesweiten Förderkontext nicht um einzelne E-LearningMaßnahmen in einzelnen Fächern, sondern um schlüssig miteinander verzahnte, hochschulweite bzw. -übergreifende Maßnahmen zum Lehren und Lernen mit digitalen und - im Sinne Döbeli Honeggers (2016), übertragen auf die Lehrerbildung - über und trotz digitale(r) Medien, auch unter Berücksichtigung informatischer Kompetenzen, die in ein Gesamtkonzept zur Digitalisierung in der Lehrerbildung strukturell einzubetten sind.

In einem ersten Schritt sollten u.E. - auch vor dem Hintergrund der oben skizzierten Desiderata und Entwicklungsnotwendigkeiten der Lehrerausbildung in Deutschland insbesondere auch unter Berücksichtigung des besonderen Mehrwertes einer Nutzung von Synergien von Verbundprojekten an mehreren Standorten solche Vorhaben gefördert werden, die Digitalisierung zum Lerngegenstand in der Lehrerbildung machen und dabei

1) eine wissenschaftlich fundierte, auch forschungsbasierte Entwicklung und systematische Verankerung digitalisierungsbezogener Kompetenzen (u.a. technisch/informatisch, didaktisch, unterrichtspraktisch, schulentwicklungsbezogen, auch unter Berücksichtigung von Einstellungen und Haltungen) im Studienverlauf verpflichtend vorsehen und dabei den kontinuierlichen und kumulativen Kompetenzaufbau innerhalb der Fächer bzw. beruflichen Fachrichtungen sowie zwischen den Fächern im Sinn der Vernetzung der fachdidaktischen, fachwissenschaftlichen und bildungswissenschaftlichen Studienanteile sowie zwischen der ersten und zweiten Phase der Lehrerausbildung kohärent stärken; 
2) Lernkontexte so gestalten, dass sie zur innovativen Praxisintegration und Handlungsorientierung im Bereich der Digitalisierung an den Schnittstellen der unterschiedlichen Ausbildungsphasen der Lehrerbildung beitragen und die Theorie-PraxisVerknüpfung systematisch befördern, so dass eine phasenübergreifende Verzahnung der Lehramtsausbildung entlang der Lehrerbildungskette durch Digitalisierung erreicht werden kann;

3) durch Digitalisierung neue Formen und Strukturen der Organisation von Lehrerbildung auch unter hochschuldidaktischen und mediendidaktischen Gesichtspunkten entwickeln.

In einem Gesamtkonzept - mit einer Vision für die künftige Entwicklung im Bereich der Digitalisierung in der Lehrerbildung - sollte deutlich dargelegt werden, welche Ziele mit welchen Maßnahmen zur Digitalisierung von Lehren und Lernen in der Lehrerbildung überprüfbar in definierten Zeiträumen erreicht werden sollen und wie der Kompetenzaufbau und die Praxisintegration und mit welchen hochschuldidaktischen und mediendidaktischen Modellen wie auch fachdidaktischen Ansätzen angelegt und in ihrer Wirkung überprüft werden. Dabei sollte im Hinblick auf die Transferierbarkeit der Entwicklungen im Regelfall die Idee leitend sein, Inhalte so zu erstellen, dass sie als Open Educational Resources (OER) frei zugänglich gemacht und über Lehrerausbildungsstandorte hinweg geteilt werden können.

Neben der Programmfinanzierung bedarf es zudem der nachhaltigen Unterstützung der neuen Aufgaben, die dauerhaft im Kontext der Digitalisierung - nicht nur im Rahmen der Lehrerbildung - entstehen bzw. zusätzlicher Ressourcen bedürfen, etwa im Bereich der Wartung von Infrastruktur, der technischen und (hochschul-)didaktischen Beratung sowie der Personalentwicklung. Schließlich soll und darf der Fokus nicht allein auf der ersten Phase der Lehrerbildung liegen; auch die Referendar*innen im Vorbereitungsdienst sowie die in der Schule bereits tätigen Lehrkräfte müssen mit überzeugenden Konzepten erreicht werden, die es systematisch zu entwickeln und zu fördern gilt.

\section{Literatur und Internetquellen}

Bertelsmann Stiftung, CHE Centrum für Hochschulentwicklung, Deutsche Telekom Stiftung \& Stifterverband für die Deutsche Wissenschaft (Hrsg.). (2018). Lehramtsstudium in der digitalen Welt - Professionelle Vorbereitung auf den Unterricht mit digitalen Medien?! Eine Sonderpublikation aus dem Projekt Monitor Lehrerbildung. Zugriff am 01.11.0218. Verfügbar unter: https://www.monitor-lehrerbildung.de/export/sites/de fault/.content/Downloads/Broschuere_Lehrerbildung-in-der-digitalen-Welt.pdf.

Bos, W., Eickelmann, B., Gerick, J., Goldhammer, F., Schaumburg, H., Schwippert, K., Senkbeil, M., Schulz-Zander, R., \& Wendt, H. (Hrsg.). (2014). ICILS 2013. Computerund informationsbezogene Kompetenzen von Schülerinnen und Schülern in der 8. Jahrgangsstufe im internationalen Vergleich. Münster: Waxmann. 
Brümmer, F., Durdel, A., Fischer-Münnich, C., Fittkau, J., Weiger, W., \& Altrichter, H. (2018). Qualitätsoffensive Lehrerbildung. Zwischenbericht der Evaluation. Hamburg: Ramboll Management Consulting GmbH. Zugriff am 01.11.2018. Verfügbar unter: https://de.ramboll.com/media/rde/2018_qlb_zwischenbericht.

Bundesregierung (2018). Ein neuer Aufbruch für Europa. Eine neue Dynamik für Deutschland. Ein neuer Zusammenhalt für unser Land. Koalitionsvertrag zwischen CDU, CSU und SPD. 19. Legislaturperiode. Zugriff am 01.11.2018. Verfügbar unter: https:// www.bundesregierung.de/breg-de/themen/koalitionsvertrag-zwischen-cdu-csu-undspd-195906.

Cress, U., Diethelm, I., Eickelmann, B., Köller, O., Nickolaus, R., Pant, H.A., \& Reiss, K. (2018). Schule in der digitalen Transformation. Perspektiven der Bildungswissenschaften. München: Deutsche Akademie der Technikwissenschaften (acatech). Zugriff am 02.11.2018. Verfügbar unter: https://www.acatech.de/wp-content/uploads/2018/10/ Schule_in-der_digitalen_Transformation_Web-1.pdf.

Döbeli Honegger, B. (2016). Mehr als 0 und 1. Schule in einer digitalisierten Welt (2. Aufl.). Bern: hep.

Eickelmann, B. (2018). Schulen und Lehrerbildung in der digitalen Welt - Thesen zwischen Hype und Zukunftsfähigkeit. Zeitschrift für Bildungsverwaltung, 39 (2), 63-72.

Eickelmann, B., \& Gerick, J. (2017). Lehren und Lernen mit digitalen Medien - Zielsetzungen, Rahmenbedingungen und Implikationen für die Schulentwicklung. Schulmanagement Handbuch, 164 (4), 54-81.

Eickelmann, B., Lorenz, R., \& Endberg, M. (2016). Die eingeschätzte Relevanz der Phasen der Lehrerausbildung hinsichtlich der Vermittlung didaktischer und methodischer Kompetenzen von Lehrpersonen für den schulischen Einsatz digitaler Medien in Deutschland und im Bundesländervergleich. In W. Bos, R. Lorenz, M. Endberg, B. Eickelmann, R. Kammerl \& S. Welling (Hrsg.), Schule digital - der Länderindikator 2016. Kompetenzen von Lehrpersonen der Sekundarstufe I im Umgang mit digitalen Medien im Bundesländervergleich (S. 149-182). Münster et al.: Waxmann.

Europäische Union (2017). Europäischer Rahmen für die Digitale Kompetenz Lehrender (DigCompEdu). Zugriff am 01.11.2018. Verfügbar unter: https://ec.europa.eu/jrc/sites/ jrcsh/files/digcompedu_leaflet_de_2018-01.pdf.

Forschungsgruppe Lehrerbildung Digitaler Campus Bayern (2017). Kernkompetenzen von Lehrkräften für das Unterrichten in einer digitalisierten Welt. Medien + Erziehung, 61 (4), 65-74.

Friedrich, S. (2017). Bildung in der digitalen Welt. Anmerkungen zum Strategiepapier der KMK. LOG IN, (187/188), 10-17.

Geissler, K.A. (1985). Lernen in Seminargruppen. Studienbrief 3 des Fernstudiums Erziehungswissenschaft „Pädagogisch-psychologische Grundlagen für das Lernen in Gruppen“. Tübingen: DIFF.

KMK (Ständige Konferenz der Kultusminister der Länder in der Bundesrepublik Deutschland) (2016). Bildung in der digitalen Welt. Strategie der Kultusministerkonferenz. Zugriff am 01.11.2018. Verfügbar unter: https://www.kmk.org/themen/bil dung-in-der-digitalen-welt/strategie-bildung-in-der-digitalen-welt.html.

KMK (Ständige Konferenz der Kultusminister der Länder in der Bundesrepublik Deutschland) (2018). Standards für die Bildungswissenschaft. Beschluss der Kultusministerkonferenz vom 16.12.2004 i.d.F. vom 12.06.2014; Überarbeitungsstand vom 09.11.2018.

Scheiter, K. (2017). Lernen mit digitalen Medien - Potenziale und Herausforderungen aus Sicht der Lehr-Lernforschung. Schulmanagement Handbuch, 164 (4), 33-47.

Schelhowe, H., Grafe, S., Herzig, B., Koubek, J., Niesyto, H., vom Berg, A., Coy, W., Hagel, H., Hasebrook, J., Kiesel, K., Reinmann, G., \& Schäfer, M. (2009). Kompetenzen in einer 
Isabell van Ackeren et al.

digital geprägten Kultur. Medienbildung für die Persönlichkeitsentwicklung, für die gesellschaftliche Teilhabe und für die Entwicklung von Ausbildungs- und Erwerbsfähigkeit. Bericht der Expertenkommission des BMBF zur Medienbildung. Zugriff am 01.11.2018. Verfügbar unter: https://www.dlr.de/pt/Portaldata/45/Resources/a_doku mente/bildungsforschung/Expertenkommission_Maerz_2009.pdf.

Schiefner-Rohs, M., Bergemann, A., Brinkmann, B., Doerr, D., Jorzik, B., Ladel, S., Scheiter, K., Schneider, R., Steinl, V., van Ackeren, I., Winter, E., \& Streitenberger, E. (2018). Lehrerinnen- und Lehrerbildung für die digitale Zukunft. Synergie - Fachmagazin für Digitalisierung in der Lehre, (6), 48-55. Zugriff am 27.11.2018. Verfügbar unter: https://uhh.de/wqrtm.

Stalder, F. (2018). Herausforderungen der Digitalität jenseits der Technologie. Synergie Fachmagazin für Digitalisierung in der Lehre, (5), 8-15. Zugriff am 31.07.2018. Verfügbar unter: https://www.synergie.uni-hamburg.de/de/media/ausgabe05/synergie05beitrag01-stalder.pdf.

Isabell van Ackeren, Prof. Dr., geb. 1974, Professorin für Bildungssystem- und Schulentwicklungsforschung, Fakultät für Bildungswissenschaften, sowie Prorektorin für Studium und Lehre an der Universität Duisburg-Essen.

Anschrift: Universität Duisburg-Essen, Universitätsstraße 2, 45141 Essen

E-Mail: isabell.van-ackeren@uni-due.de

Stefan Aufenanger, Prof. i. R. Dr., geb. 1950, war Professor für Erziehungswissenschaft und Medienpädagogik an der Universität Mainz.

Anschrift: Hegelstr. 54, 55122 Mainz

E-Mail: stefan@aufenanger.de

Birgit Eickelmann, Prof. Dr., geb. 1971, Professorin für Schulpädagogik an der Universität Paderborn.

Anschrift: Universität Paderborn, Warburger Str. 100, 33098 Paderborn

E-Mail: birgit.eickelmann@upb.de

Steffen Friedrich, Prof. i.R. Dr. habil., geb. 1951, war Professor für Didaktik der Informatik an der TU Dresden.

Anschrift: TU Dresden, Fakultät Informatik, 01062 Dresden

E-Mail: steffen.friedrich@tu-dresden.de

Rudolf Kammerl, Prof. Dr., geb. 1971, Professor für Pädagogik mit dem Schwerpunkt Medienpädagogik an der Friedrich-Alexander-Universität Erlangen-Nürnberg.

Anschrift: Friedrich-Alexander-Universität Erlangen-Nürnberg, Institut für Erziehungswissenschaft, Regensburger Straße 160, 90478 Nürnberg

E-Mail: rudolf.kammerl@fau.de

Julia Knopf, Prof. Dr., geb. 1980, Geschäftsführende Leitung des Forschungsinstituts Bildung Digital und Professorin für Fachdidaktik Deutsch Primarstufe an der Universität des Saarlandes.

Anschrift: Universität des Saarlandes, Campus C6 3, 66123 Saarbrücken

E-Mail: julia.knopf@mx.uni-saarland.de 
Kerstin Mayrberger, Prof. Dr., geb. 1977, Professorin für Lehren und Lernen an der Hochschule mit dem Schwerpunkt Mediendidaktik an der Universität Hamburg. Anschrift: Universität Hamburg, Schlüterstraße 51, 20146 Hamburg

E-Mail: kerstin.mayrberger@uni-hamburg.de

Heike Scheika, M.A., geb. 1964, Landessprecherin des Bundesarbeitskreises der Seminar- und Fachleiter*innen e. V., stellvertretende Gesamtseminarleiterin am Staatlichen Studienseminar für Lehrerausbildung Gera, Leiterin des Staatlichen Studienseminars für das Lehramt an Grundschulen.

Anschrift: BAK Lehrerbildung, Auf dem Scheitberg 45, 07589 Bocka

E-Mail: scheika.bak.thueringen@web.de

Katharina Scheiter, Prof. Dr., geb. 1974, Professorin für Empirische Lehr-Lernforschung an der Eberhard Karls Universität Tübingen und Leiterin der Arbeitsgruppe Multiple Repräsentationen am Leibniz-Institut für Wissensmedien.

Anschrift: Leibniz-Institut für Wissensmedien, Schleichstr. 6, 72076 Tübingen

E-Mail: k.scheiter@iwm-tuebingen.de

Mandy Schiefner-Rohs, JProf. Dr., geb. 1980, Juniorprofessorin für Pädagogik mit Schwerpunkt Schulentwicklung im Fachbereich Sozialwissenschaften an der TU Kaiserslautern.

Anschrift: TU Kaiserslautern, Erwin-Schrödinger-Straße, 67663 Kaiserslautern

E-Mail: mandy.rohs@sowi.uni-kl.de 